\title{
Naturally derived scaffolds for dental pulp regeneration:
}

\section{A review}

\author{
Zul Faizuddin Osman ${ }^{1}$, Azlina Ahmad ${ }^{1}$, Khairul Bariah Ahmad Amin Noordin ${ }^{1}$ \\ (1) Universiti Sains Malaysia, School of Dental Sciences, Kubang Kerian, Malaysia
}

Date submitted:

Nov 18, 2018

Date accepted:

Jan 25, 2019

Online publication date:

June 15, 2019

\section{Corresponding Author: \\ Dr Khairul Bariah Ahmad Amin \\ Noordin, \\ Universiti Sains Malaysia, \\ School of Dental Sciences, \\ Kubang Kerian, Malaysia \\ kbariah@usm.my}

\begin{abstract}
Dental pulp regeneration via stem cell engineering approach offers a great potential in tackling the difficulty in dental clinical situations such as teeth loss and periodontal diseases. One of the potential approaches in dental tissue engineering includes the application of dental stem cells-based therapy. In this approach, dental stem cells are generally cultured on special biodegradable membrane filters or scaffolds, allowing for three-dimensional (3D) pulp tissues formation before it can be inserted into the area of damaged dental pulp for dentin regeneration. The naturally derived scaffold, onto which stem cells are let to grow include platelet rich fibrin (PRF), collagen, hyaluronic acid (HA), polysaccharides, silk and amniotic membrane (AM). Biological scaffolds offer special characteristics such as biocompatibility, biodegradability, low immunogenicity and ability to support cells growth for various applications of tissue engineering. This review discusses the available naturally derived scaffolds for dental pulp regeneration and highlights their potential advantages. We also aim to improve the understanding of the application of natural scaffolds in dental pulp regeneration.
\end{abstract}

Keywords: Biological scaffolds, dental pulp regeneration, amniotic membrane, stem cells differentiation, tissue engineering, natural scaffolds.

\section{Introduction}

Regenerative medicine is an interdisciplinary approach involving the utilization of live cells to repair, replace, or restore the normal function of damaged human tissues and organs (1). Tremendous advancements in this field have been made since Leland Kaiser first introduced the term in 1992 (2). One that holds great potential in this field is stem cell engineering, which could be the future of regenerative medicine.

Difficult clinical dentistry situations such as teeth loss and periodontal diseases could be alleviated using stem cell engineering, as it isolates readily accessible post-natal stem cells from the teeth and provides a non-invasive way of harvesting stem cells, especially from teeth meant for extraction. The mesenchymal stem cell (MSC) is one of the widely known types of stem cells that have been found suitable for regenerative medicine due to its high proliferation and multi-potential capabilities. There are many MSC which originated from the teeth, and they are named according on the site of the teeth where they are harvested, includes dental pulp stem cells (DPSCs), stem cells from human exfoliated deciduous teeth (SHED), periodontal lig- ament stem cells (PDLSCs), dental follicle progenitor stem cells (DFPCs), and stem cells from apical papilla (SCAPs) (3). In stem cells and tissue engineering, the scaffold is a supporting matrix onto which the stem cells and tissue grow. To ensure the successful growth of the cell and its differentiation, materials from the scaffold must be able to interact with the host tissue and provide an ideal environment to support the tissue growth.

\section{Dental pulp regeneration}

The dental pulp is a complex structure comprising connective tissue, MSCs, neural fibers, blood vessels, and the lymphatic system. It plays a role in producing dentin and serves to support the biological and physiological vitality of the dentin (4). Various types of cells exist in the pulp tissue, including immune cells, fibroblasts, MSCs, vascular cells, and nerve cells. It has been established that odontoblasts play important roles in dentinogenesis. Dentin plays a significant role in the formation of teeth. Dentin regeneration occurs during primary and secondary dentinogenesis (5).

Important aspects of pulp regeneration include the vascularization of tissues, the formation of neurons, and deposition of 
dentin (6). An ideal scaffold for dental pulp regeneration should allow direct contact between the restorative material and the pulp. It has to meet three criteria: first, biocompatibility of the scaffold; second, the material must be stiff to withstand repeated masticatory pressure; and third, the material should provide a tight seal against the dentinal wall to prevent infiltration of oral microorganisms into the pulp chamber (7). This complex and dynamic environment associated with the dental pulp is maintained in a delicate balance, with odontoblasts supporting the mineralized tissues. At the same time, other cell types are positioned to support the activity of the odontoblasts. For dental pulp tissue maintenance and repair, a native population of MSCs provides a reservoir of pluripotent cells with the capacity of differentiation into a variety of cells as required. The long-term function of the whole tooth depends on the progenitor cells. Therefore, progenitor cells are an important consideration when designing scaffolds for dental pulp regeneration (8).

\section{Types of scaffolding for tissue engineering in dental pulp regeneration}

Damaged teeth are commonly attributed to caries infection or traumatic injury. In cases of irreversible damage, it is critical to develop regeneration modalities to restore dental pulp integrity and functions. Stem cells, scaffolds, and growth factors are essential to dental pulp tissue regeneration. During the regeneration processes, growth factors induce proliferation and differentiation of stem cells into specific cells for tissue defects, and scaffolds provide support for cell growth, differentiation, and formation of tissue. This approach may allow for regeneration of the entire tooth, local regeneration of the dental pulp in the dentin defect area from the residual dental pulp, or regeneration of the dental pulp from an apical dental pulp or periapical tissues, including the periodontal ligament and bone (9).

In regenerative endodontics, any type of scaffold should have a 3D structure that can support cell organization and vascularization (10). The goal of dental pulp regeneration revolves around rebuilding dental material, as well as regenerating periodontal ligament, maxillofacial, and oral bones. In order to achieve successful regeneration, the scaffolds, cells, and bioactive signaling molecules are required to induce stem cell differentiation into the dental tissue type (11).

An ideal scaffold should be able to interact with cells, offer a platform for cell adhesion and proliferation, and enhance the regeneration of tissue (12). Early studies on dental pulp tissue engineering with tooth slices or segments have used polylactic or polyglycolic acid (PLGA) as scaffold materials. Subsequently, various other types of scaffolds, including naturally derived scaffolds such as alginate, collagen, and fibrin or synthetic scaffolds such as PLGA, polyethylene glycol (PEG), or self-assembling peptide (SAP), have been explored (13).

Synthetic scaffolds are widely accepted as platforms for dental pulp regeneration applications because they can be customized and optimized (14). However, it is challenging to design synthetic scaffolds that closely mimic the functions of native tissues. Therefore, naturally derived scaffolds are believed to provide a better platform for dental regeneration in clinical situations.

\section{Naturally derived scaffolds}

Naturally derived scaffolds can be produced from components found in the extracellular matrix (ECM), such as collagen, fibrinogen, and HA. Natural scaffolds have desirable charac- teristics such as biocompatibility, low immunogenicity, biodegradability, and mechanical properties similar to natural tissue. Natural polymers, such as collagen, protein, chitosan, silk, alginate, $\mathrm{HA}$, and their derivatives, were among the first materials used as scaffolds for tissue restoration and regeneration (14). In this review, we highlight several naturally derived scaffolds for dental pulp tissue regeneration, such as PRF, collagen, and $\mathrm{HA}$, as well as polysaccharides such as alginate, chitosan and cellulose, silk, and AM. Table 1 shows the list of the key studies using natural scaffolds for dentin-pulp regeneration.

\subsection{Platelet-Rich Fibrin}

PRF is a natural fibrin-based scaffold rich with platelets and growth factors. It is a natural concentrate prepared from an anti-coagulant-free blood harvest (15), without any addition of biochemical compounds. PRF requires simple preparation. The PRF concentrate produces a strong natural fibrin matrix, which shows a unique architecture suitable for wound healing and regeneration (16).

The material has immune capacity (17) and important cytokines (16) as well as growth factors, which can accelerate the regeneration process. The presence of physiological thrombin in PRF creates equilateral junctions in polymerized fibrin, which results in the buffered release of growth factors (up to 28 days) and a flexible fibrin network, an appropriate microenvironment for cell migration. Thus, PRF is a preferable scaffold in regenerative endodontics and is initially used to accelerate tissue healing (17).

Chen et al. (18) studied the cytobiological effects of PRF on DPSCs and investigated the potential regenerative capacity of a novel PRF tissue-engineered transplant. They found that PRF induced the proliferation of canine DPSCs in a dose- and time-dependent manner. It also assisted in the differentiation of DPSCs to odontoblast or osteoblast by up-regulating the expression of the odontoblast markers and bone sialoprotein (BSP) gene. Transplantation of the PRF scaffold cultured with DPSCs helped in the regeneration of homogeneous and compact pulp-like tissues with abundantly distributed blood vessels, and the deposition of regenerated dentin along the intracanal walls at 8 weeks post-operation. PRF scaffolds might serve as a potential therapy in regenerative endodontics for pulp revitalization or revascularization (18).

A study was conducted by integrating PRF into fibrin glue to enrich the microenvironment with growth factors. The study showed promising results when transplanted with dental pulp cells. An animal was used to develop a complete tooth with crown, root, pulp, enamel, dentin, odontoblast, cementum, blood vessels, and periodontal ligaments in indiscriminate shape. The study demonstrated that dental pulp cells seeded into fibrin glue-PRF were able to regenerate a complete tooth (19). Studies have also shown that PRF is more superior compared to collagen as a scaffold for human periosteal cell proliferation. In addition, rapid healing of the wound was observed without pain, dryness, or discharging complications (16).

\subsection{Collagen}

Collagen is a natural component in the dental pulp with collagen type 1 being the most used collagen type used. Collagen-based scaffolds is widely used in dental regeneration (20). Collagen scaffolds have been shown to provide a suitable environment for pulp progenitor and adult stem cells with odontoblastic properties in vitro, and adequate new tissue develop- 
Table 1: List of the key studies using natural scaffolds for dentin pulp regeneration

\begin{tabular}{|c|c|c|}
\hline Natural scaffold & Study by & Results \\
\hline \multirow[t]{4}{*}{ PRF } & Ji et al., 2014 (15) & $\begin{array}{l}\text { Stimulates proliferation and mineralization of PLDCs in vitro and regenera- } \\
\text { tion of tooth-periodontal ligament-alveolar interfaces in vivo }\end{array}$ \\
\hline & Zhang et al., 2017 (16) & $\begin{array}{l}\text { Similar expression of cytokines in fresh and lyophilized PRF which able to } \\
\text { promote cells proliferation and differentiation }\end{array}$ \\
\hline & Bakhtiar et al., 2017 (17) & Successful regeneration of pulpal contents in immature teeth \\
\hline & Yang et al., 2012 (18) & DPSCs differentiated to complete tooth in vivo \\
\hline \multirow[t]{2}{*}{ Collagen } & Yang et al., 2012 (19) & $\begin{array}{l}\text { Induced high proliferation and odontoblastic differentiation of DPSCs in vitro } \\
\text { and in vivo when enriched with BMP-7 }\end{array}$ \\
\hline & Soares et al., 2016 (20) & $\begin{array}{l}\text { Induced cell proliferation and odontoblastic capacity of DPSCs when inte- } \\
\text { grated with calcium aluminate }\end{array}$ \\
\hline \multirow[t]{2}{*}{$\mathrm{HA}$} & Leong et al., 2016 (21) & $\begin{array}{l}\text { Regeneration of dental pulp tissue with angiogenesis and neurogenesis in } \\
\text { vivo }\end{array}$ \\
\hline & Chrepa et al., 2017 (22) & Induced high odontoblastic markers and posses better cell viability \\
\hline
\end{tabular}

\begin{tabular}{|c|c|c|}
\hline \multirow[t]{4}{*}{ Alginate } & Colombo et al., 2014 (8) & Induced odontoblast-like cells differentiation when treated with TGF $\beta 1$ \\
\hline & Kumabe et al., 2006 (23) & Induced odontoblastic markers and differentiation to odontoblast-like cells \\
\hline & Fujiwara et al., 2006 (24) & $\begin{array}{l}\text { Induced differentiation of rat dental pulp cells into odontoblast-like cells and } \\
\text { stimulates calcification }\end{array}$ \\
\hline & Yu et el., 2017 (25) & Induced proliferation and adhesion of human dental pulp cells \\
\hline
\end{tabular}

$\begin{array}{lll}\text { Chitosan } & \text { Amir et al., } 2014(26) & \text { Increased macaque DPSCs proliferation, ALP activity and mineralization } \\ \text { Li et al., } 2014(27) & \begin{array}{l}\text { Induced repair mechanism of dental pulp when applied to exposed pulp } \\ \text { tissue on canine }\end{array}\end{array}$

$\begin{array}{lll}\text { Cellulose } & \text { Teti et al., } 2015(28) & \begin{array}{l}\text { Induced adhesion, cell viability, odontogenic and osteogenic markers of } \\ \text { DPSCs }\end{array}\end{array}$

\begin{tabular}{lll} 
Silk & Yang et al., $2015(29)$ & Induced DPSCs viability and generated pulp-like tissue structure \\
Woloszyk et al., $2015(30)$ & Induced mineralization of DPSCs and support dental pulp regeneration \\
\hline AM & Alshehadat et al., 2016 (31) & $\begin{array}{l}\text { Promoted DPSCs attachment, proliferation and differentiation without any } \\
\text { notable inflammatory responses }\end{array}$ \\
\hline
\end{tabular}

ment with low immunogenic reactions in vivo (21). However, collagen mechanical properties are weak and easily degrade in biological media. Therefore, there are many studies that have instead used a construct of the collagen scaffold composite integrated with other materials for dental pulp regeneration.
Yang et al. (22) investigated the feasibility and efficacy of a chitosan/collagen human bone morphogenetic protein-7 (BMP7)-enriched scaffold using DPSCs for in vitro and in vivo studies. The in vitro results indicate that DPSCs on the gene-activated scaffold demonstrated higher proliferation rates and 
odontoblastic differentiation behaviors compared to cells on the pure scaffolds. The subcutaneous in vivo implanted chitosan/ collagen BMP-7-enriched scaffold showed up-regulated expression of dentin sialophosphoprotein (DSPP) compared to the pure scaffold groups. Therefore, the chitosan/collagen-enriched BMP-7 scaffold appears to be an effective candidate for gene delivery; it also enhanced DPSCs differentiation for odontoblast-like phenotype in vitro and in vivo (22).

Besides that, a chitosan-collagen scaffold integrated with calcium-aluminate mineral was found compatible with HDPCs. The findings showed that cell proliferation was associated with increased odontoblastic differentiation capability (23). Another study showed that a fibroblast growth factor-2 (FGF2)-enriched collagen hydrogel scaffold has excellent biocompatibility in periodontal tissue-engineering applications, including periodontal attachment re-organization (24). Cells seeded onto collagen scaffolds implanted in animals for in vivo pulp regeneration demonstrated regeneration of pulp tissue with angiogenesis and neurogenesis. Interestingly, a combination of collagen and a synthetic scaffold, the PLGA scaffold, showed a regenerative capacity of swine DPSCs after replantation into the animal teeth (21).

\subsection{Hyaluronic Acid}

$\mathrm{HA}$ and collagen, which represent the main structural components of ECM for several tissues, have been extensively tested for regenerative endodontic applications (25). HA and its derivatives are known to have excellent potential for tissue engineering because HA can be chemically and structurally modified for various applications (14). HA is also one of the glycosaminoglycans in ECM and plays important roles in maintaining morphologic organization by preserving extracellular spaces (26).

In addition, the HA sponge has the appropriate physical structure, biocompatibility, and biodegradability to work as an implant for dental pulp regeneration (14). However, combinations of growth factors with the HA sponge are needed for the development of restorative treatment of dental pulps with sound dentin. According to Gathani and Raghavendra (26), other advantages of $\mathrm{HA}$ include its ability to assist in the differentiation of dental MSCs to odontoblasts, contribution to the formation of dentin matrix and dental pulp, its bioactive, non-immunogenic, and non-thrombogenic properties, and its beneficial role in wound healing. The HA can also be used as an injectable scaffold.

Chrepa et al. (27) demonstrated Restylane, an FDA-approved HA-based injectable gel, as having promising potential as a scaffold for regenerative endodontic procedures. In their study, SCAPs were cultured either alone or in mixtures with either Restylane or Matrigel scaffolds. It was shown that the HA-based scaffold possessed better cell viability compared to the Matrigel and control. Restylane also had significantly higher ALP activity and promoted high expression of the odontoblastic markers, DSPP and DMP-1, when compared to the control. These results indicate for the first time that an FDA-approved product can be used as a promising scaffold for regenerative endodontic procedures and that it may, along with other factors, lead to dental pulp regeneration (27).

\subsection{Polysaccharides}

Polysaccharide scaffolds are characterized by high-porosity materials, which provide a great surface for seeding cells for tissue repair, regeneration, or remodeling. It has several characteristics that could make it an excellent scaffold. Some examples of polysaccharides used in tissue engineering are alginate, chitosan, and cellulose. Polysaccharides have been applied in various forms and have been tested in the engineering of several types of tissues including dental, blood vessels, bone, cartilage, neural tissue, and others (28).

\subsubsection{Alginate}

Alginate is a natural polysaccharide derived from brown seaweed and has good characteristics as a scaffold such as low immunogenicity, biocompatibility, and mildness of gelation conditions (14). Alginate hydrogels are compatible with MSCs derived from the gingiva and periodontal ligament, and, when loaded with TGF $\beta 1$, were found to induce odontoblast-like cell differentiation in a human tooth slice model (8).

Kumabe et al. (29) observed expression of DSPP mRNA coding dentin sialoprotein (DSP) when beta-glycerophosphate was added to a culture medium. An increase in alkaline phosphatase (ALP), an early marker for odontoblast differentiation, was also demonstrated. The study showed that subcultured dental pulp cells actively differentiated into odontoblast-like cells and induced calcification in the alginate scaffold. In another study by Fujiwara et al. (30), an alginate scaffold seeded with rat dental pulp cells and HDPCs was implanted in the back of nude mice. The findings indicate that the seeded cells differentiated into odontoblast-like cells and stimulated calcification.

Alginate hydrogel scaffolds are relatively stable and can retain high levels of biological activity to promote the proliferation of HDPCs. Various shapes and sizes of the scaffolds can be prepared to suit the needs of dental pulp regeneration. It is also known that, by seeding cells at a high concentration, better cell adhesion to the scaffold materials can be promoted (31).

\subsubsection{Chitosan}

Chitosan is a chitin-derived biopolymer, a nitrogen-containing polysaccharide produced by glucose. Chitosan is biocompatible, flexible, and possesses antibacterial properties that accelerate wound healing (32). It can be shaped into various structures, including microspheres, paste, sponges, and porous scaffolds with promising potential in tissue engineering (33). Its similarity with glycosaminoglycan, its good biocompatibility with pulp cells, and good antimicrobial activity are interesting features that lend well to dentin regeneration.

Amir et al. (34) showed that a chitosan scaffold was capable of increasing cell proliferation, ALP activity, and mineralized matrix deposition on DPSCs in vitro. The chitosan scaffold induced the repair mechanism of a dental pulp when applied to the exposed pulp tissue on canine teeth without any tissue necrosis observed (35). Chitosan-collagen scaffolds have been extensively studied in mineralized tissue regeneration due to their similarity to bone/dentin ECM, controlled degradation rate, and mechanical strength (36).

\subsubsection{Cellulose}

Cellulose is the most abundant and renewable biopolymer in nature. Scientists in the fields of tissue engineering and regenerative medicine have shown increasing interest in cellulose scaffolds because of their low density and excellent biodegradability associated with low risks of ecological toxicity (37). The biocompatibility and low water solubility of cellulose allow for good control over scaffold design. In vivo applications of cel- 
lulose-based materials have also demonstrated insignificant inflammatory response reactions (38). Moreover, it has good mechanical properties because of the strong hydrogen bonding between the cellulose chains (39).

In a study by $\mathrm{He}$ et al. (38), a cellulose nanofiber scaffold was produced using a rotating collector with a water coagulation bath. The nanofiber scaffold exhibited a distinct and uniform fiber texture, which makes it as an excellent platform for human dental follicle stem cell attachment and proliferation on the entire scaffold. Furthermore, Teti et al. (40) observed good adhesion and viability in cells cultured on a cellulose-hydroxyapatite hydrogel composite compared to cells cultured on a carboxymethyl cellulose hydrogel only. The former showed up-regulation of osteogenic and odontogenic markers in DPSCs.

Additionally, bacteria-derived cellulose has been established as a versatile biomaterial. According to Acasigua et al. (41), integration of HA acid and gelatin in bacterial cellulose scaffolds showed higher cell adhesion and proper cell distribution over time. Taken together, cellulose-based scaffolds could be considered as a promising candidate for dental pulp complex and periodontal tissue engineering.

\subsection{Silk scaffold}

The domesticated mulberry silkworm Bombyx mori produces a silk fibroin (SF) natural polymer. Silk scaffolds are biocompatible and non-toxic, easy to process, have superior mechanical properties, controlled degradability, oxygen and water permeability, and the ability to promote cell attachment and proliferation (36). Additionally, SF is less immunogenic and anti-inflammatory, compared with either PLGA or collagen scaffolds, making it a popular scaffold candidate for the regeneration of cartilage, skin, bone, blood vessel, nerve, or ligament (42).

Recent evidence has shown that the silk fibroin scaffold is suitable for dental pulp tissue regeneration, especially in 3D structures of the scaffold compared to the 2D silk film, where higher cells adhesion were observed. A study showed that the hexafluoroisopropanol (HFIP)-based silk scaffold is more superior in assisting with soft dental pulp formation when compared to the aqueous-based silk scaffold. HFIP provides slower material degradation and better support. Dental pulp cells seeded on HFIP silk scaffolds have promoted the formation of mineralized tissue, indicating the usefulness of these scaffolds in osteodentin formation (36).

Yang et al. (42) assessed the potential of freeze-dried porous silk fibroin scaffolds seeded with DPSCs and treated with basic FGF (bFGF) for dental pulp regeneration in an ectopic root canal transplantation model. They found that the bFGF-incorporated scaffold promoted DPSC viability and generated a pulp-like tissue structure. Histologically, the generated tissue was well vascularized, with new matrix deposition and dentin-like tissue formation, and consisted of both the transplanted and host-derived cells. Collectively, these data support the use of the bFGF-treated silk fibroin scaffold as a potential scaffold candidate for future treatment concepts in regenerative endodontics, particularly for dental pulp regeneration (42). Silk scaffolds also offer good mineralization potential to support dental pulp regeneration and regenerative endodontic therapy approaches (43).

\subsection{Amniotic membrane (AM)}

The AM structure consists of a single epithelial layer with two distinctive parts, a thick basal membrane and a stromal side, which is avascular (44). Besides having low immunogenicity and good mechanical properties, AM also releases many types of biological properties such as anti-inflammatory, anti-microbial, anti-fibrosis, and anti-scarring characteristics (45).

Preserved human AM is a novel tissue engineered biomaterial with great potential in the field of medicine and dentistry, particularly for the regeneration of lost tissues and accelerated repair (46). Moreover, the potential of AM as a scaffold has gained great interest in many types of research focusing on clinical periodontology application (47) and dental pulp regeneration (28).

Various components in the scaffold such as collagen, laminin, fibronectin and vitronectin in the AM basal membrane ECM play important roles in the attachment and growth of a cell to a scaffold. ECM molecules also serve as adhesion ligands, transmitting signals via interaction at the cell surface receptors. Cells seeded on the AM scaffold can penetrate into the porous structure of the AM scaffold and are also highly interconnected (47).

In oral and periodontal surgeries, AM was observed to reduce scarring and inflammation besides enhancing wound healing. It could also serve as a scaffold for proliferation and differentiation of cells, owing to its antimicrobial properties. Moreover, ECM and its components such as growth factors, provide an excellent biomaterial to act as a native scaffold for tissue engineering. For large-scale application, AM is known to be reproducible, easily procured, processed, and transported (48).

According to Chopra and Thomas (46), AM biological features such as rapid re-epithelialization, vascularization, and formation of granulation tissue makes it a good biological dressing. The size of the AM can be customized based on the recipient-desired site. It can be cut, folded, and transplanted without compromising the cultured cells on the membrane. AM also has also shown promising ability to prevent damage during surgical practices; thus increasing the success rate of the procedures (49).

AM is widely used to assist osteogenic differentiation. One study by Chen et al. (50) showed that both the basal and stromal sides of the accellular AM were able to provide a suitable matrix for osteogenic differentiation of dental apical papilla cells (APCs) with stem cell characteristics. Interestingly, a recent study done by Alshehadat (28) showed that AM provided a suitable environment for dental pulp stem cell attachment, and proliferation and differentiation into odontoblast cells without any notable inflammatory response.

\section{Conclusion}

An important achievement in the field of regenerative medicine is the ability to regenerate teeth rather than replace them. Rapid development in the field of stem cell-based therapies has shown great promise in the treatment of dental diseases due to the massive involvement of stem cells in dental pulp regeneration. In tissue engineering approaches for dental pulp regeneration, stem cells can be grown on scaffolds that function to mechanically support the cells' biological actions for ultimate tissue replacement.

Naturally derived scaffolds offer great advantages due to their unique features such as biocompatibility, biodegradability, and low immunogenicity, and the ability to support stem cell growth and differentiation. They are suitable for dental implan- 
tation, and, are therefore favorable for tissue engineering applications. Next-generation naturally derived scaffolds in combination with cells and therapeutic agents such as antibiotics, enzymes, growth factors, and DNAs, can successfully be incorporated in dental pulp regeneration strategies. This will enable a customized element to be specifically designed, fabricated, and applied to achieve the desired therapeutic outcome.

In addition to feasibility and technical concerns, process development is a significant hurdle that must be managed. It includes standardization and consistency of scaffolds in experimental and clinical setups. Nevertheless, data from experimental procedures can be utilized for the process development of the scaffolds such that they will be ready for commercialization. Moreover, the efficiency of the scaffolds in supporting dental pulp regeneration in in vitro and in vivo experiments and translating this to clinical practice is pivotal in ensuring the success of this approach.

\section{Acknowledgments}

Zul Faizuddin Osman and Khairul Bariah Ahmad Amin Noordin were involved in the conception and design of the manuscript. Khairul Bariah Ahmad Amin Noordin and Azlina Ahmad were involved in the critical revision of the manuscript for important intellectual content. This review was supported by the Universiti Sains Malaysia Research University Grant (1001/ PPSG/812127).

\section{Conflict of interest}

The authors declare that they have nothing to disclose regarding conflict of interest with respect to this manuscript.

\section{References}

1. Chien KR. Regenerative medicine and human models of human disease. Nature. 2008;453(7193):302-325.

2. Kaiser $L$. The future of multihospital systems. Topics in health care financing. 1992;18(4):32-45.

3. Ledesma-Martínez E, Mendoza-Núñez VM, Santiago-Osorio E. Mesenchymal Stem Cells Derived from Dental Pulp: A Review. Stem cells international. $2015 ; 2016$.

4. Tatullo M, Marrelli M, Shakesheff KM, White LJ. Dental pulp stem cells: function, isolation and applications in regenerative medicine. Journal of tissue engineering and regenerative medicine. 2015;9(11):1205-1216.

5. Arana-Chavez VE, Massa LF. Odontoblasts: the cells forming and maintaining dentine. The international journal of biochemistry \& cell biology. 2004;36(8):1367-1373.

6. Yang J-w, Zhang Y-f, Wan C-y, et al. Autophagy in SDF-1a-mediated DPSC migration and pulp regeneration. Biomaterials. 2015;44:11-23.

7. Cao $\mathrm{Y}$, Song $\mathrm{M}, \mathrm{Kim} \mathrm{E}$, et al. Pulp-dentin regeneration: current state and future prospects. Journal of dental research. 2015;94(11):1544-1551.

8. Colombo JS, Moore AN, Hartgerink JD, D'Souza RN. Scaffolds to control inflammation and facilitate dental pulp regeneration. Journal of endodontics. 2014;40(4):612.

9. Kitamura C, Nishihara T, Terashita M, Tabata Y, Washio A. Local regeneration of dentin-pulp complex using con- trolled release of fgf-2 and naturally derived sponge-like scaffolds. International journal of dentistry. 2012;2012.

10. Murray PE, Garcia-Godoy F, Hargreaves KM. Regenerative endodontics: a review of current status and a call for action. Journal of endodontics. 2007;33(4):377-390.

11. Nakashima M. Bone morphogenetic proteins in dentin regeneration for potential use in endodontic therapy. Cytokine \& growth factor reviews. 2005;16(3):369-376.

12. Khojasteh A, Motamedian SR, Rad MR, Shahriari MH, Nadjmi N. Polymeric vs hydroxyapatite-based scaffolds on dental pulp stem cell proliferation and differentiation. World journal of stem cells. 2015;7(10):1215-1221.

13. Galler KM, Brandl FP, Kirchhof S, et al. Suitability of different natural and synthetic biomaterials for dental pulp tissue engineering. Tissue Engineering Part $A$. 2017;24(3-4):234-244.

14. Zhang L, Morsi Y, Wang Y, Li Y, Ramakrishna S. Review scaffold design and stem cells for tooth regeneration. Japanese Dental Science Review. 2013;49(1):14-26.

15. Ji B, Sheng L, Chen G, et al. The combination use of platelet-rich fibrin and treated dentin matrix for tooth root regeneration by cell homing. Tissue Engineering Part $A$. 2014;21(1-2):26-34.

16. Zhang J, Qi X, Luo X, Li D, Wang H, Li T. Clinical and immunohistochemical performance of lyophilized platelet-rich fibrin (Ly-PRF) on tissue regeneration. Clinical Implant Dentistry and Related Research. 2017;19(3):466477.

17. Bakhtiar H, Esmaeili S, Tabatabayi SF, Ellini MR, Nekoofar $\mathrm{MH}$, Dummer PM. Second-generation platelet concentrate (platelet-rich fibrin) as a scaffold in regenerative endodontics: a case series. Journal of endodontics. 2017;43(3):401-408.

18. Chen Y-J, Zhao Y-H, Zhao Y-J, et al. Potential dental pulp revascularization and odonto-/osteogenic capacity of a novel transplant combined with dental pulp stem cells and platelet-rich fibrin. Cell Tissue Res. 2015;361(2):439455.

19. Yang $\mathrm{KC}$, Wang $\mathrm{CH}$, Chang $\mathrm{HH}$, Chan WP, Chi $\mathrm{CH}$, Kuo TF. Fibrin glue mixed with platelet-rich fibrin as a scaffold seeded with dental bud cells for tooth regeneration. Journal of tissue engineering and regenerative medicine. 2012;6(10):777-785.

20. Piva E, Silva AF, Nör JE. Functionalized scaffolds to control dental pulp stem cell fate. Journal of endodontics. 2014;40(4):33-40.

21. Leong DJX, Setzer FC, Trope M, Karabucak B. Biocompatibility of two experimental scaffolds for regenerative endodontics. Restorative dentistry \& endodontics. 2016;41(2):98-105.

22. Yang X, Han G, Pang X, Fan M. Chitosan/collagen scaffold containing bone morphogenetic protein-7 DNA supports dental pulp stem cell differentiation in vitro and in vivo. Journal of biomedical materials research Part $A$. 2012.

23. Soares DG, Rosseto HL, Basso FG, Scheffel DS, Hebling J, Costa CA. Chitosan-collagen biomembrane 
embedded with calcium-aluminate enhances dentinogenic potential of pulp cells. Brazilian oral research. 2016;30(1).

24. Momose $\mathrm{T}$, Miyaji $\mathrm{H}$, Kato $\mathrm{A}$, et al. Collagen Hydrogel Scaffold and Fibroblast Growth Factor-2 Accelerate Periodontal Healing of Class II Furcation Defects in Dog. The open dentistry journal. 2016;10:347-359.

25. Panseri S, Montesi M, Dozio SM, Savini E, Tampieri A Sandri M. Biomimetic scaffold with aligned Microporosity Designed for Dentin regeneration. Frontiers in bioengineering and biotechnology. 2016;4

26. Gathani KM, Raghavendra SS. Scaffolds in regenerative endodontics: A review. Dental research journal. 2016;13(5):379-386.

27. Chrepa V, Austah O, Diogenes A. Evaluation of a Commercially Available Hyaluronic Acid Hydrogel (Restylane) as Injectable Scaffold for Dental Pulp Regeneration: An In Vitro Evaluation. Journal of endodontics. 2017;43(2):257-262.

28. Alshehadat SA, Thu HA, Hamid SSA, Nurul AA, Rani SA, Ahmad A. Scaffolds for dental pulp tissue regeneration: A review. Int Dent Med J Adv Res. 2016;2:1-12.

29. Kumabe S, Nakatsuka M, Kim G-S, et al. Human dental pulp cell culture and cell transplantation with an alginate scaffold. Okajimas folia anatomica Japonica. 2006;82(4):147-156

30. Fujiwara S, Kumabe S, Iwai Y. Isolated rat dental pulp cell culture and transplantation with an alginate scaffold Okajimas folia anatomica Japonica. 2006;83(1):15-24.

31. Yu H, Ma D, Wu B. Gelatin/alginate hydrogel scaffolds prepared by $3 \mathrm{D}$ bioprinting promotes cell adhesion and proliferation of human dental pulp cells in vitro. Nan fang yi ke da xue xue bao= Journal of Southern Medical University. 2017;37(5):668-672.

32. Mota J, Yu N, Caridade SG, et al. Chitosan/bioactive glass nanoparticle composite membranes for periodontal regeneration. Acta Biomaterialia. 2012;8(11):41734180 .

33. Jazayeri HE, Fahmy MD, Razavi M, et al. Dental Applications of Natural-Origin Polymers in Hard and Soft Tissue Engineering. Journal of Prosthodontics. 2016;25(6):510517.

34. Amir LR, Suniarti DF, Utami S, Abbas B. Chitosan as a potential osteogenic factor compared with dexamethasone in cultured macaque dental pulp stromal cells. Cell Tissue Res. 2014;358(2):407-415.

35. Li F, Liu X, Zhao S, Wu H, Xu HH. Porous chitosan bilayer membrane containing TGF- $\beta 1$ loaded microspheres for pulp capping and reparative dentin formation in a dog model. Dental Materials. 2014;30(2):172-181.

36. Sharma S, Srivastava D, Grover S, Sharma V. Biomaterials in tooth tissue engineering: a review. Journal of clinical and diagnostic research: JCDR. 2014;8(1):309-315.

37. Domingues RM, Gomes ME, Reis RL. The potential of cellulose nanocrystals in tissue engineering strategies Biomacromolecules. 2014;15(7):2327-2346.
38. He X, Cheng L, Zhang X, Xiao Q, Zhang W, Lu C. Tissue engineering scaffolds electrospun from cotton cellulose. Carbohydrate polymers. 2015;115:485-493.

39. Petrauskaite $\mathrm{O}$, Gomes PdS, Fernandes $\mathrm{MH}$, et al. Biomimetic mineralization on a macroporous cellulose-based matrix for bone regeneration. BioMed research international. 2013;2013.

40. Teti G, Salvatore V, Focaroli S, et al. In vitro osteogenic and odontogenic differentiation of human dental pulp stem cells seeded on carboxymethyl cellulose-hydroxyapatite hybrid hydrogel. Frontiers in physiology. 2015;6.

41. Acasigua X, Arisoly G, Olyveira GMd, et al. Novel Chemically Modified Bacterial Cellulose Nanocomposite as Potential Biomaterial for Stem Cell Therapy Applications. Current Stem Cell Research \& Therapy. 2014:117-123.

42. Yang J-w, Zhang Y-f, Sun Z-y, Song G-t, Chen Z. Dental pulp tissue engineering with bFGF-incorporated silk fibroin scaffolds. Journal of biomaterials applications. 2015;30(2):221-229.

43. Woloszyk A, Dircksen SH, Bostanci N, Müller R, Hofmann S, Mitsiadis TA. Influence of the mechanical environment on the engineering of mineralised tissues using human dental pulp stem cells and silk fibroin scaffolds. Plos one. 2014;9(10):e111010.

44. Díaz-Prado S, Muiños-López E, Hermida-Gómez T, et al. Human amniotic membrane as an alternative source of stem cells for regenerative medicine. Differentiation. 2011;81(3):162-171.

45. Niknejad H, Peirovi H, Jorjani M, Ahmadiani A, Ghanavi J, Seifalian AM. Properties of the amniotic membrane for potential use in tissue engineering. Eur Cells Mater. 2008;15:88-99.

46. Chopra A, Thomas BS. Amniotic membrane: A novel material for regeneration and repair. Biomimetics Biomaterials and Tissue Engineering. 2013;18(1):1-8.

47. Mohan R, Bajaj A, Gundappa M. Human amnion membrane: Potential applications in oral and periodontal field. Journal of International Society of Preventive \& Community Dentistry. 2017;7(1):15.

48. Gupta A, Kedige SD, Jain K. Amnion and chorion membranes: potential stem cell reservoir with wide applications in periodontics. International journal of biomaterials. $2015 ; 2015$.

49. Iwasaki K, Komaki M, Yokoyama N, et al. Periodontal regeneration using periodontal ligament stem cell-transferred amnion. Tissue Engineering Part A. 2013;20(34):693-704

50. Chen Y-J, Chung M-C, Yao C-CJ, et al. The effects of acellular amniotic membrane matrix on osteogenic differentiation and ERK1/2 signaling in human dental apical papilla cells. Biomaterials. 2012;33(2):455-463. 